\title{
Stability Optimization of a Disc Brake System with Hybrid Uncertainties for Squeal Reduction
}

\author{
Hui Lü and Dejie Yu \\ State Key Laboratory of Advanced Design and Manufacturing for Vehicle Body, Hunan University, Changsha, Hunan 410082, China \\ Correspondence should be addressed to Dejie Yu; djyu@hnu.edu.cn
}

Received 2 November 2015; Revised 28 January 2016; Accepted 28 January 2016

Academic Editor: Tai Thai

Copyright (C) 2016 H. Lü and D. Yu. This is an open access article distributed under the Creative Commons Attribution License, which permits unrestricted use, distribution, and reproduction in any medium, provided the original work is properly cited.

\begin{abstract}
A hybrid uncertain model is introduced to deal with the uncertainties existing in a disc brake system in this paper. By the hybrid uncertain model, the uncertain parameters of the brake with enough sampling data are treated as probabilistic variables, while the uncertain parameters with limited data are treated as interval probabilistic variables whose distribution parameters are expressed as interval variables. Based on the hybrid uncertain model, the reliability-based design optimization (RBDO) of a disc brake with hybrid uncertainties is proposed to explore the optimal design for squeal reduction. In the optimization, the surrogate model of the real part of domain unstable eigenvalue of the brake system is established, and the upper bound of its expectation is adopted as the optimization objective. The lower bounds of the functions related to system stability, the mass, and the stiffness of design component are adopted as the optimization constraints. The combinational algorithm of Genetic Algorithm and Monte-Carlo method is employed to perform the optimization. The results of a numerical example demonstrate the effectiveness of the proposed optimization on improving system stability and reducing squeal propensity of a disc brake under hybrid uncertainties.
\end{abstract}

\section{Introduction}

Brake squeal is a noise problem caused by self-frictioninduced vibrations which can induce dynamic instabilities of brake systems [1]. Poor stability performance of a disc brake system could lead to an inconvenient squealing noise. This frequently leads to customer complaints and results in enormous warranty costs. Therefore, extensive efforts have been undertaken by industrial corporations as well as by the scientific communities to resolve the squeal problem. Early researches on brake squeal are mostly dedicated to the root causes of brake squeal and several representative mechanisms have been attributed to brake squeal phenomenon, such as stick-slip, sprag-slip, hammering, mode-coupling, and time delay [2-5]. Moreover, several comprehensive review papers can be found on the researches of brake squeal [6-10]. Of those mechanisms, the mode-coupling has attracted the most attention in these literatures. However, the root cause of brake squeal is still not fully understood due to its complexity.

The optimization designs of disc brake systems for squeal reduction have received many researchers' attentions recently. For example, Guan et al. [11] explored sensitivity analysis methods to determine the dominant modal parameters of the substructures of a brake system for squeal suppression, and the dominant modal parameters were set as the target of optimization design to seek the modifications of the disc and the bracket to eliminate the squeal mode. Spelsberg-Korspeter $[12,13]$ performed a structural optimization of brake rotors to emphasize the potential of designing rotor geometry robust against self-excited vibrations, and the mathematical difficulties of such an optimization were discussed. Lakkam and Koetniyom [14] proposed an optimization study on brake squeal aimed at minimizing the strain energy of vibrating pads with constrained layer damping; the results of this research could be a guide to specify the position of the constrained layer damping patch under pressure conditions. Shintani and Azegami [15] presented a solution to a nonparametric shape optimization problem of a disc brake model to suppress squeal noise; the optimum shape of the pad was found and the real part of the complex eigenvalue representing the cause of brake squeal was minimized.

The abovementioned studies on structural optimization of disc brake systems are restricted to deterministic optimization, in which all design variables and parameters involved 
are regarded certain. In practical engineering, uncertain factors widely exist in the structures and systems, as well as in the brake systems. Under uncertain cases, the optimum obtained from a deterministic optimization could easily violate the constraints and cause unreliable solutions and designs [1618]. In order to take into account various uncertainties, the RBDO is introduced and has been intensively studied both in the methodology and in applications [19-21]. Compared with the deterministic optimization, the RBDO aims to seek a reliable optimum by converting the deterministic constraints into probabilistic constraints. Therefore, the RBDO can be considered as the potential method to improve the stability performance of the uncertain disc brake systems for squeal reduction.

Due to the effects of manufacturing errors, aggressive environment factors, wear and unpredictable external excitations, uncertainties associated with material properties, geometric dimensions, and contact conditions are unavoidable. On the investigation of disc brake squeal considering uncertainties, just only a few research papers have been published at present. Chittepu [22] has carried out a robustness evaluation of a brake system with a stochastic model, in which the material scatter was modeled by random variables and the geometrical tolerances were modeled by random fields. Based on the polynomial chaos expansions, the stochastic eigenvalue problems and the stability of a simplified disc brake have been investigated by Sarrouy et al. [23], in which the friction coefficient and the contact stiffness of the disc brake system were modeled as random parameters. Tison et al. [24] have proposed a complete strategy to improve the prediction of brake squeal by introducing uncertainty and robustness concepts into the simulations. The strategy mainly relied on the integration of complex eigenvalue calculations, probabilistic analysis, and a robustness criterion. Lü and $\mathrm{Yu}$ $[25,26]$ have proposed practical approaches for the stability analysis and optimization of disc brakes with probabilistic uncertainties or interval uncertainties for squeal reduction, in which an uncertain parameter is treated as either probabilistic variable or interval variable. It can be seen that the investigations on uncertain brake squeal problem are not still comprehensive currently. It is necessary to develop more different uncertain methods for the uncertain researches on disc brake squeal reduction.

It is well known that probabilistic methods are the traditional approach to cope with these uncertainties arising in practical engineering problems, just as we can see in the abovementioned studies [22-24]. In the probabilistic methods, the uncertain parameters are treated as probabilistic variables whose probability distributions are defined unambiguously [27]. To construct the precise probability distributions of probabilistic variables, a large amount of statistical information or experimental data is required. Unfortunately, in the design stage of a disc brake system, the information to construct the precise probability distributions of some probabilistic variables (e.g., the friction coefficient or the thicknesses of wearing components) is not always sufficient due to the immeasurability or wear effects. In the last two decades, the interval probabilistic model has been proposed for overcoming the deficiencies of probabilistic methods. In the interval probabilistic model, the uncertain parameters are treated as interval probabilistic variables whose distribution parameters with limited information are expressed as interval variables instead of precise values. The interval probabilistic model was firstly proposed by Elishakoff et al. [28, 29] and subsequently applied to the structural response analysis [30] and the structural reliability analysis [31]. From the overall perspective, researches on the interval probabilistic model are still in the preliminary stage and some important issues are still unsolved. For example, the application of the interval probabilistic model in the stability analysis of disc brakes is not yet explored.

The purpose of this paper is to take into account the hybrid uncertainties existing in a disc brake and develop an effective optimization for improving its system stability and suppressing its squeal propensity. For this purpose, a RBDO approach based on the hybrid uncertain model is proposed. In the hybrid uncertain model, the uncertainties of the thickness of nonwear component, the mass densities and elastic modulus of component materials, and the brake pressure are represented by using probabilistic variables, whose distribution parameters are well defined, whereas the uncertainties of the friction coefficient, the thickness of disc, and friction material are modeled by interval probabilistic variables, whose distribution parameters are expressed as interval variables. To facilitate computation, the response surface (RS) model is used to replace the time-consuming FE simulations. Based on the methods of RSM, CEA, and hybrid uncertain analysis, the RBDO model for improving the system stability of the disc brake is constructed. In the RBDO model, the upper bound of the expectation of the real part of the domain unstable eigenvalue is selected as design objective, while the lower bounds of the functions related to system stability, the mass and the stiffness of design component, are adopted as optimization constraints. The effectiveness of the proposed optimization is demonstrated by a numerical example.

The rest of this paper is organized as follows: Section 2 introduces the CEA of disc brakes; Section 3 describes the construction of the RBDO of a disc brake with hybrid uncertainties; Section 4 presents the procedure of the proposed RBDO; Section 5 provides a numerical example of the application of the proposed optimization, and finally Section 6 draws the major conclusions.

\section{Complex Eigenvalue Analysis of Disc Brake}

2.1. A Simplified Disc Brake. For the purpose of simulating the vibration characteristics of a brake system reasonably with an acceptable computation, a simplified disc brake system is taken for investigation. The simplified brake consists of a disc and a pair of brake pads, as shown in Figure 1. The disc is rigidly mounted on the axle hub and therefore rotates with the wheel. The pair of brake pad assemblies, which consist of the friction materials and the back plates, is pressed against the disc in order to generate a frictional torque to slow the rotation of the disc. The similar simplified model has been previously considered and successfully used by some studies, such as $[23,32-34]$. 


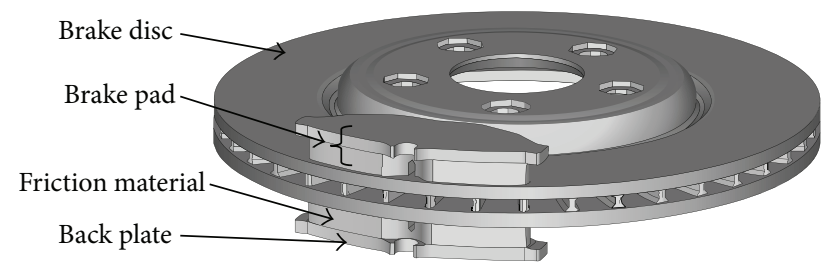

Figure 1: A simplified model of a disc brake system.

2.2. Complex Eigenvalue Analysis. The CEA can be carried out by two stages. In the first stage, the steady state of the brake system is found and in the second stage, the CEA is performed.

In the steady state of the brake system, the vector of applied forces $\mathbf{F}(\dot{\mathbf{y}}, \mathbf{y})$ is balanced by the vector of nonlinear stiffness forces $\mathbf{F}_{k}(\mathbf{y})$. It can be expressed as

$$
\mathbf{F}_{k}(\mathbf{y})=\mathbf{F}(\dot{\mathbf{y}}, \mathbf{y}),
$$

where $\mathbf{y}$ is the steady-state displacement vector and $\dot{\mathbf{y}}$ is the first derivative of $\mathbf{y}$ with respect to time. The nonlinearity of stiffness forces $\mathbf{F}_{k}(\mathbf{y})$ is mainly due to the contact formulation, in which the contact stiffness is commonly added at the disc/pad interface. The disc is about one hundred times stiffer than the frictional material which is a mixture of many components; thus deformation of a pad under loading is much greater and nonlinearity behavior occurs. For a more complete discussion of system nonlinearities, the readers can refer to [35].

After the steady state of the system is found, in the second stage, the stability of system is assessed. The extended nonlinear equation of motion including the mass matrix $\mathbf{M}$ and the damping matrix $\mathbf{D}$ is given by

$$
\mathbf{M y}+\mathbf{D} \dot{\mathbf{y}}+\mathbf{F}_{k}(\mathbf{y})=\mathbf{F}(\dot{\mathbf{y}}, \mathbf{y}),
$$

where $\ddot{y}$ is the second derivative of $\mathbf{y}$ with respect to time. The system can be linearized about the steady state

$$
\mathbf{y}=\mathbf{y}_{0}+\Delta \mathbf{y}
$$

where $\mathbf{y}_{0}$ is the steady-state displacement vector and $\Delta \mathbf{y}$ denotes small perturbation in the vicinity of the steady state. After linearization, the resulting equation is given by

$$
\mathbf{M} \cdot \Delta \ddot{\mathbf{y}}+\mathbf{D} \cdot \Delta \dot{\mathbf{y}}+\mathbf{K} \cdot \Delta \mathbf{y}=\mathbf{H}_{1} \cdot \Delta \dot{\mathbf{y}}+\mathbf{H}_{0} \cdot \Delta \mathbf{y},
$$

where matrices $\mathbf{H}_{0}$ and $\mathbf{H}_{1}$ are the partial derivatives of $\mathbf{F}(\dot{\mathbf{y}}, \mathbf{y})$ with respect to the displacements and the velocities, respectively. Equation (4) can be simplified to

$$
\mathbf{M} \cdot \Delta \ddot{\mathbf{y}}+\widehat{\mathbf{D}} \cdot \Delta \dot{\mathbf{y}}+\widehat{\mathbf{K}} \cdot \Delta \mathbf{y}=\mathbf{0},
$$

where $\widehat{\mathbf{D}}$ and $\widehat{\mathbf{K}}$ are the new damping matrix and stiffness matrix; then the complex eigenvalue problem can be formulated as

$$
\left(\mathbf{M} \lambda^{2}+\widehat{\mathbf{D}} \lambda+\widehat{\mathbf{K}}\right) \phi=\mathbf{0},
$$

where $\lambda$ is the complex eigenvalue and $\phi$ is the complex eigenmode. The complex eigenvalues of the system can be obtained by solving the complex eigenvalue problem of (6). The $i$ th complex eigenvalue $\lambda_{i}$ can be expressed as

$$
\lambda_{i}=\alpha_{i}+j \beta_{i},
$$

where $\alpha_{i}$ and $\beta_{i}$ are the real and imaginary parts of the complex eigenvalue $\lambda_{i}$, respectively.

It is widely known that a vibration system will become unstable if the real part of a complex eigenvalue of the system is positive. Therefore, the positive real parts of the eigenvalues can be taken as an indicator for system stability and squeal propensity. The main aim of this study is to minimize the positive real part of the dominant unstable eigenvalue of the brake system.

It is worth mentioning that the damping is not taken into account in the current study. Just as the recent study [36] points out, the damping models of friction materials or other components have not been extensively studied at present and there is no reliable data on the damping of brake systems. In most cases, the damping can stabilize brake systems and excluding it will provide more potential unstable eigenvalues. Thus, industrial researchers tend to exclude the damping and set a target value for the real part or the damping ratio of an unstable eigenvalue in CEA of practical engineering. If the real part or the damping ratio is not larger than the target value, the brake system will be considered as stable and acceptable [36]. However, two main effects of damping on coalescence patterns have been noticed in fact, namely, the shifting effect and the smoothing effect [37]. The first one always stabilizes the brake, whereas the second one may cause instability. For more detailed and extensive discussion of damping on system stability, the readers can refer to [37].

Moreover, the main limitation of CEA is the use of a linear model which excludes dynamic contact. Sinou et al. $[38,39]$ pointed out that CEA may lead to underestimation or overestimation of the unstable modes of a brake. In [38], it was shown that additional unstable modes appeared during transient oscillations, which were not predicted by CEA. Thus, the nonlinear methods have been proposed and developed to approximate the transient behavior in the time domain [40], or the limit cycle of frictional systems subjected to selfexcited vibration and squeal noise in the frequency domain [41]. Besides, the stochastic studies have been recently carried out to determine the limit cycles of a self-excited nonlinear system with friction which is commonly used to represent brake squeal phenomenon [42]. However, we mainly focus our attention on the stability analysis and optimization of a linear brake system with uncertainties in this work, considering that CEA is a fast and efficient strategy for an industrial finite element model. Therefore, just the CEA approach is used in this paper.

\section{RBDO of the Disc Brake System with Hybrid Uncertainties}

3.1. Surrogate Model Based on RMS. In engineering design, the coupling of optimization algorithm with finite element 
analysis (FEA) may be inefficient, since the iterative analyses during optimization usually require enormous iterations and high computational cost, especially for RBDO problem. As a result, the surrogate models have been widely adopted in engineering applications to alleviate the computational burden. By surrogate models, the explicit mathematical relationship between functional responses and design variables can be established with a moderate number of FEA runs. RS model is one of the simplest and most popular surrogate models, which can be treated as an effective alternative to FEA and has been widely adopted in design optimization [43].

Mathematically, by the RS model, the real part of a complex eigenvalue of FEA can be defined in terms of basis function as

$$
\alpha(\mathbf{x})=\sum_{j=1}^{N} a_{j} \varphi_{j}(\mathbf{x}),
$$

where $\alpha(\mathbf{x})$ is the real part of a complex eigenvalue, $\varphi_{j}(\mathbf{x})$ are the basis functions, $N$ is the number of basis functions, and $a_{j}$ represents the regression coefficients. When using a quadratic model, the full set of the second-order polynomials of $\varphi_{j}(\mathbf{x})$ is given as

$$
1, x_{1}, x_{2}, \ldots, x_{n}, x_{1}^{2}, x_{1} x_{2}, \ldots, x_{1} x_{n}, \ldots, x_{n}^{2}
$$

and the surrogate model of $\alpha(\mathbf{x})$ could be thus defined as

$$
\alpha(\mathbf{x})=a_{0}+\sum_{i=1}^{n} a_{i} x_{i}+\sum_{i j(i<j)} a_{i j} x_{i} x_{j}+\sum_{i=1}^{n} a_{i i} x_{i}^{2},
$$

where $\mathbf{x}=\left[x_{1}, x_{2}, \ldots, x_{n}\right]^{T}$ denotes the variable vector that determines the response $\alpha(\mathbf{x})$ and $a_{0}, a_{i}, a_{i j}$, and $a_{i i}$ are the estimated regression coefficients which can be obtained from the design of experiment (DOE) and by the least square method (LSM) [44]. The cross product terms $x_{i} x_{j}$ represent the two-variable interactions and the square terms $x_{i}^{2}$ represent the second-order nonlinearities. $n$ is the number of the variables.

After the RS model is established, its accuracy should be assessed. It is necessary to conduct an analysis of variance (ANOVA) to test the model, so as to ensure its fitting accuracy and significance [45].

It is worth mentioning that the surrogate model with kriging predictor has been recently proposed to investigate the propensity of brake squeal by Nobari et al. [36] and Nechak et al. [46]. However, the current study is not focused on the surrogate models; thus just the most widely used surrogate model (RS model) is applied here. Indeed, it has great significance to carry out further studies on the application of different surrogate models to squeal problem in the future.

3.2. Disc Brake System with Hybrid Uncertainties. Due to the complex working environment and operating conditions, the occurrence of brake squeal is intermittent or perhaps even casual in general, and it is difficult to be captured and reproduced artificially. This is possibly related to the uncertainties existing in the brake system. For instance, the friction coefficient is changed in the course of braking. This characteristic of friction coefficient makes it possible to provide the energy source for brake squeal [9]. So it will be more reasonable and be of great significance, if the uncertainties are taken into account for the stability analysis of the disc brake systems.

For the disc brake system, uncertainties in material, geometry, and loading properties and manufacturing errors are unavoidable. Usually, the most common approach to model the uncertainties is the probabilistic model. By this approach, $\alpha(\mathbf{x})$ in (10) for an uncertain optimization problem can be generally expressed as

$$
\alpha(\mathbf{x})=\alpha\left(\mathbf{x}_{D}, \mathbf{x}_{P}\right)
$$

where $\mathbf{x}_{D}=\left[x_{D, 1}, x_{D, 2}, \ldots, x_{D, k}\right]^{T}$ is the design variable vector and $k$ is the number of design variables; $\mathbf{x}_{P}=$ $\left[x_{P, 1}, x_{P, 2}, \ldots, x_{P, l}\right]^{T}$ is the probabilistic variable vector which is taken as nondesign variables and $l$ is the number of probabilistic variables.

In the real case, the disc brake is a rather complex system which is related to frictional contact and wear [47]. It is difficult or even impossible to obtain the precise values of the distribution parameters of some probabilistic variables, such as the friction coefficient and the thickness of wearing components. In order to model the uncertainties of these parameters more properly, these uncertain parameters are treated as interval probabilistic variables whose distribution parameters are expressed as interval variables rather than unique values in this study. In this case, $\alpha(\mathbf{x})$ in (11) then becomes an interval probabilistic objective function for a hybrid uncertain optimization problem and can be expressed as

$$
\alpha(\mathbf{x})=\alpha\left(\mathbf{x}_{D}, \mathbf{x}_{P}\right)=\alpha\left(\mathbf{x}_{D}, \mathbf{x}_{P 1}, \mathbf{x}_{P 2}\right),
$$

where $\mathbf{x}_{P 1}=\left[x_{P 1,1}, x_{P 1,2}, \ldots, x_{P 1, l-m}\right]^{T}$ is the probabilistic variable vector of the hybrid uncertain problem and $l-m$ is the number of probabilistic variables. $\mathbf{x}_{P 2}=\left[x_{P 2,1}, x_{P 2,2}, \ldots\right.$, $\left.x_{P 2, m}\right]^{T}$ is the interval probabilistic variables vector of the hybrid uncertain problem, and $m$ is the number of interval probabilistic variables.

Due to the manufacturing or measuring errors, the actual values of the design variable $\mathbf{x}_{D}$ may deviate from their theoretical values in engineering practice. In this case, the design variables $\mathbf{x}_{D}$ can be treated as the probabilistic variables as well.

\subsection{RBDO of the Disc Brake System with Hybrid Uncertainties.} The RBDO model of the disc brake system with hybrid uncertainties can be generally expressed as

$$
\begin{array}{ll}
\min _{\mathbf{x}_{D}} & \alpha(\mathbf{x})=\alpha\left(\mathbf{x}_{D}, \mathbf{x}_{P 1}, \mathbf{x}_{P 2}\right) \\
\text { s.t. } & P\left(g_{r}\left(\mathbf{x}_{D}, \mathbf{x}_{P 1}, \mathbf{x}_{P 2}\right) \geq 0\right) \geq \eta_{r}, \quad r=1,2, \ldots, t \\
& \mathbf{x}_{D \text {,lower }} \leq \mathbf{x}_{D} \leq \mathbf{x}_{D \text {,upper }},
\end{array}
$$

where $\alpha\left(\mathbf{x}_{D}, \mathbf{x}_{P 1}, \mathbf{x}_{P 2}\right)$ is the interval probabilistic objective function. $g_{r}\left(\mathbf{x}_{D}, \mathbf{x}_{P 1}, \mathbf{x}_{P 2}\right)$ is the $r$ th limit-state function, and $t$ is the number of limit-state functions. $g_{r}\left(\mathbf{x}_{D}, \mathbf{x}_{P 1}, \mathbf{x}_{P 2}\right)=0$ is the limit-state equation by which the designspace is divided 
into the safe region $g_{r}\left(\mathbf{x}_{D}, \mathbf{x}_{P 1}, \mathbf{x}_{P 2}\right)>0$ and the failure region $g_{r}\left(\mathbf{x}_{D}, \mathbf{x}_{P 1}, \mathbf{x}_{P 2}\right)<0 . P\left(g_{r}\left(\mathbf{x}_{D}, \mathbf{x}_{P 1}, \mathbf{x}_{P 2}\right) \geq 0\right)$ is the probability for $g_{r}\left(\mathbf{x}_{D}, \mathbf{x}_{P 1}, \mathbf{x}_{P 2}\right) \geq 0 . \eta_{r}$ is the $r$ th system reliability index which is close to 1 with the increase of required reliability level.

The objective function $\alpha\left(\mathbf{x}_{D}, \mathbf{x}_{P 1}, \mathbf{x}_{P 2}\right)$ is an interval probabilistic function. Without considering the interval variables existing in the interval probabilistic variables $\mathbf{x}_{P 2}$ temporarily, the objective function $\alpha\left(\mathbf{x}_{D}, \mathbf{x}_{P 1}, \mathbf{x}_{P 2}\right)$ can be treated as a pure probabilistic function. The probabilistic characteristic of the values of $\alpha\left(\mathbf{x}_{D}, \mathbf{x}_{P 1}, \mathbf{x}_{P 2}\right)$ can be expressed as the expectation of $\alpha\left(\mathbf{x}_{D}, \mathbf{x}_{P 1}, \mathbf{x}_{P 2}\right)$. Therefore, the purpose of the RBDO model can be expressed as

$$
\min _{\mathbf{x}_{D}} \mu\left(\alpha\left(\mathbf{x}_{D}, \mathbf{x}_{P 1}, \mathbf{x}_{P 2}\right)\right)
$$

where $\mu\left(\alpha\left(\mathbf{x}_{D}, \mathbf{x}_{P 1}, \mathbf{x}_{P 2}\right)\right)$ is the expectation of $\alpha\left(\mathbf{x}_{D}, \mathbf{x}_{P 1}, \mathbf{x}_{P 2}\right)$.

As the interval variables exist in $\mathbf{x}_{P 2}$ actually, the obtained expectation $\mu\left(\alpha\left(\mathbf{x}_{D}, \mathbf{x}_{P 1}, \mathbf{x}_{P 2}\right)\right)$ is a function of interval variables. $\mu\left(\alpha\left(\mathbf{x}_{D}, \mathbf{x}_{P 1}, \mathbf{x}_{P 2}\right)\right)$ is uncertain suffering from interval variables. For the disc brake system, the optimization goal is to reduce $\mu\left(\alpha\left(\mathbf{x}_{D}, \mathbf{x}_{P 1}, \mathbf{x}_{P 2}\right)\right)$ to improve system stability. Consequently, if the maximal value of $\mu\left(\alpha\left(\mathbf{x}_{D}, \mathbf{x}_{P 1}, \mathbf{x}_{P 2}\right)\right.$ ) (marked as $\left.\mu\left(\alpha\left(\mathbf{x}_{D}, \mathbf{x}_{P 1}, \mathbf{x}_{P 2}\right)\right)_{\text {upper }}\right)$ satisfies the design requirements, the other values (which are no more than the maximal value) of $\mu\left(\alpha\left(\mathbf{x}_{D}, \mathbf{x}_{P 1}, \mathbf{x}_{P 2}\right)\right)$ will also satisfy the design requirements. As a result, the maximal value of $\mu\left(\alpha\left(\mathbf{x}_{D}, \mathbf{x}_{P 1}, \mathbf{x}_{P 2}\right)\right)$ can be selected as the optimization objective.

Therefore, the purpose of the RBDO of the disc brake system with hybrid uncertainties for squeal reduction can be expressed as

$$
\min _{\mathbf{x}_{D}} \mu\left(\alpha\left(\mathbf{x}_{D}, \mathbf{x}_{P 1}, \mathbf{x}_{P 2}\right)\right)_{\text {upper }},
$$

where $\mu\left(\alpha\left(\mathbf{x}_{D}, \mathbf{x}_{P 1}, \mathbf{x}_{P 2}\right)\right)_{\text {upper }}$ is the upper bound of $\mu\left(\alpha\left(\mathbf{x}_{D}\right.\right.$, $\left.\left.\mathbf{x}_{P 1}, \mathbf{x}_{P 2}\right)\right)$

Without considering the interval variables existing in interval probabilistic variables $\mathbf{x}_{P 2}$ temporarily, the $r$ th limitstate function $g_{r}\left(\mathbf{x}_{D}, \mathbf{x}_{P 1}, \mathbf{x}_{P 2}\right)$ can be treated as a pure probabilistic function. The probability of $g_{r}\left(\mathbf{x}_{D}, \mathbf{x}_{P 1}, \mathbf{x}_{P 2}\right) \geq 0$, namely, $P\left(g_{r}\left(\mathbf{x}_{D}, \mathbf{x}_{P 1}, \mathbf{x}_{P 2}\right) \geq 0\right)$, is a deterministic value. As the interval variables exist in $\mathbf{x}_{P 2}$ actually, $P\left(g_{r}\left(\mathbf{x}_{D}, \mathbf{x}_{P 1}, \mathbf{x}_{P 2}\right) \geq\right.$ $0)$ is a function of interval variables. $P\left(g_{r}\left(\mathbf{x}_{D}, \mathbf{x}_{P 1}, \mathbf{x}_{P 2}\right) \geq\right.$ $0)$ is uncertain suffering from interval variables, and the uncertainty of $P\left(g_{r}\left(\mathbf{x}_{D}, \mathbf{x}_{P 1}, \mathbf{x}_{P 2}\right) \geq 0\right)$ can be described by the variational range of $P\left(g_{r}\left(\mathbf{x}_{D}, \mathbf{x}_{P 1}, \mathbf{x}_{P 2}\right) \geq 0\right)$. If the lower bound of $P\left(g_{r}\left(\mathbf{x}_{D}, \mathbf{x}_{P 1}, \mathbf{x}_{P 2}\right) \geq 0\right.$ ) (marked as $\left.P\left(g_{r}\left(\mathbf{x}_{D}, \mathbf{x}_{P 1}, \mathbf{x}_{P 2}\right) \geq 0\right)_{\text {lower }}\right)$ is larger than $\eta_{r}$, the other values of $P\left(g_{r}\left(\mathbf{x}_{D}, \mathbf{x}_{P 1}, \mathbf{x}_{P 2}\right) \geq 0\right)$ within the variational range will be also larger than $\eta_{r}$. Thus, the constraint conditions can be rewritten as

$$
P\left(g_{r}\left(\mathbf{x}_{D}, \mathbf{x}_{P 1}, \mathbf{x}_{P 2}\right) \geq 0\right)_{\text {lower }} \geq \eta_{r}, \quad r=1,2, \ldots, t,
$$

where $P\left(g_{r}\left(\mathbf{x}_{D}, \mathbf{x}_{P 1}, \mathbf{x}_{P 2}\right) \geq 0\right)_{\text {lower }}$ is the lower bound of $P\left(g_{r}\left(\mathbf{x}_{D}, \mathbf{x}_{P 1}, \mathbf{x}_{P 2}\right) \geq 0\right)$.
Based on the discussion above, the RBDO of the disc brake system with hybrid uncertainties for squeal reduction can be expressed as

$$
\begin{array}{ll}
\min _{\mathbf{x}_{D}} & \mu\left(\alpha\left(\mathbf{x}_{D}, \mathbf{x}_{P 1}, \mathbf{x}_{P 2}\right)\right)_{\text {upper }} \\
\text { s.t. } & P\left(g_{r}\left(\mathbf{x}_{D}, \mathbf{x}_{P 1}, \mathbf{x}_{P 2}\right) \geq 0\right)_{\text {lower }} \geq \eta_{r},
\end{array}
$$$$
r=1,2, \ldots, t
$$

$$
\mathbf{x}_{D, \text { lower }} \leq \mathbf{x}_{D} \leq \mathbf{x}_{D, \text { upper }}
$$

3.4. Calculations of the Objective Function and Constraint Function. Based on the clear expression of the RS model $\alpha(\mathbf{x})$, the Monte-Carlo method can be considered as a potential method to calculate the objective function $\mu\left(\alpha\left(\mathbf{x}_{D}, \mathbf{x}_{P 1}\right.\right.$, $\left.\left.\mathbf{x}_{P 2}\right)\right)_{\text {upper }}$ and the constraint function $P\left(g_{r}\left(\mathbf{x}_{D}, \mathbf{x}_{P 1}, \mathbf{x}_{P 2}\right) \geq\right.$ $0)_{\text {lower. }}$. The main procedures of the calculations based on Monte-Carlo method can be described in the following.

Step 1. Generate $i$ th $\left(i=1,2, \ldots, N_{i}\right)$ sample of the interval variables existing in the interval probabilistic variable vector $\mathbf{x}_{P 2}$. Then $\mathbf{x}_{P 2}$ degenerated to the probabilistic variable vector with the sample values of the interval variables and can be marked as probabilistic variable vector $\mathbf{x}_{P 2}^{(i)}$.

Step 2. Generate $j$ th $\left(j=1,2, \ldots, N_{j}\right)$ sample of the probabilistic variable vectors $\mathbf{x}_{P 1}$ and $\mathbf{x}_{P 2}^{(i)}$. Then $\mathbf{x}_{P 1}$ and $\mathbf{x}_{P 2}^{(i)}$ degenerated to the deterministic value vector with the sample values of the probabilistic variables and can be marked as $\mathbf{x}_{P 1}^{(j)}$ and $\mathbf{x}_{P 2}^{(i, j)}$

Step 3. Calculate the values of $\alpha\left(\mathbf{x}_{D}, \mathbf{x}_{P 1}, \mathbf{x}_{P 2}\right)^{(i, j)}=\alpha\left(\mathbf{x}_{D}, \mathbf{x}_{P 1}^{(j)}\right.$, $\left.\mathbf{x}_{P 2}^{(i, j)}\right)$ and $g_{r}\left(\alpha\left(\mathbf{x}_{D}, \mathbf{x}_{P 1}, \mathbf{x}_{P 2}\right)\right)^{(i, j)}=g_{r}\left(\alpha\left(\mathbf{x}_{D}, \mathbf{x}_{P 1}^{(j)}, \mathbf{x}_{P 2}^{(i, j)}\right)\right)$.

Step 4. Has the sampling of $\mathbf{x}_{P 1}$ and $\mathbf{x}_{P 2}^{(i)}$ ended? Namely, does $j$ equate to $N_{j}$ (the number of samples of probabilistic variable vectors)? If no, $j=j+1$ and go on to Step 2 . If yes, calculate the expectation $\mu\left(\alpha\left(\mathbf{x}_{D}, \mathbf{x}_{P 1}, \mathbf{x}_{P 2}\right)\right)^{(i)}$ and the probability $P\left(g_{r}\left(\mathbf{x}_{D}, \mathbf{x}_{P 1}, \mathbf{x}_{P 2}\right) \geq 0\right)^{(i)}$ based on all values of $\alpha\left(\mathbf{x}_{D}, \mathbf{x}_{P 1}, \mathbf{x}_{P 2}\right)^{(i, j)}$ and $g_{r}\left(\alpha\left(\mathbf{x}_{D}, \mathbf{x}_{P 1}, \mathbf{x}_{P 2}\right)\right)^{(i, j)}, j=1,2, \ldots, N_{j}$, and export them.

Step 5. Has the sampling of interval variables existing in $\mathbf{x}_{P 2}$ ended? Namely, does $i$ equate to $N_{i}$ (the number of samples of interval variable vector)? If no, $i=i+1$ and go on to Step 1. If yes, select $\mu\left(\alpha\left(\mathbf{x}_{D}, \mathbf{x}_{P 1}, \mathbf{x}_{P 2}\right)\right)_{\text {upper }}$ and $P\left(g_{r}\left(\mathbf{x}_{D}, \mathbf{x}_{P 1}\right.\right.$, $\left.\left.\mathbf{x}_{P 2}\right) \geq 0\right)_{\text {lower }}$ from all values of $\mu\left(\alpha\left(\mathbf{x}_{D}, \mathbf{x}_{P 1}, \mathbf{x}_{P 2}\right)\right)^{(i)}$ and $P\left(g_{r}\left(\mathbf{x}_{D}, \mathbf{x}_{P 1}, \mathbf{x}_{P 2}\right) \geq 0\right)^{(i)}, i=1,2, \ldots, N_{i}$, and export them.

The procedure can be summarized in the flowchart shown in Figure 2. 


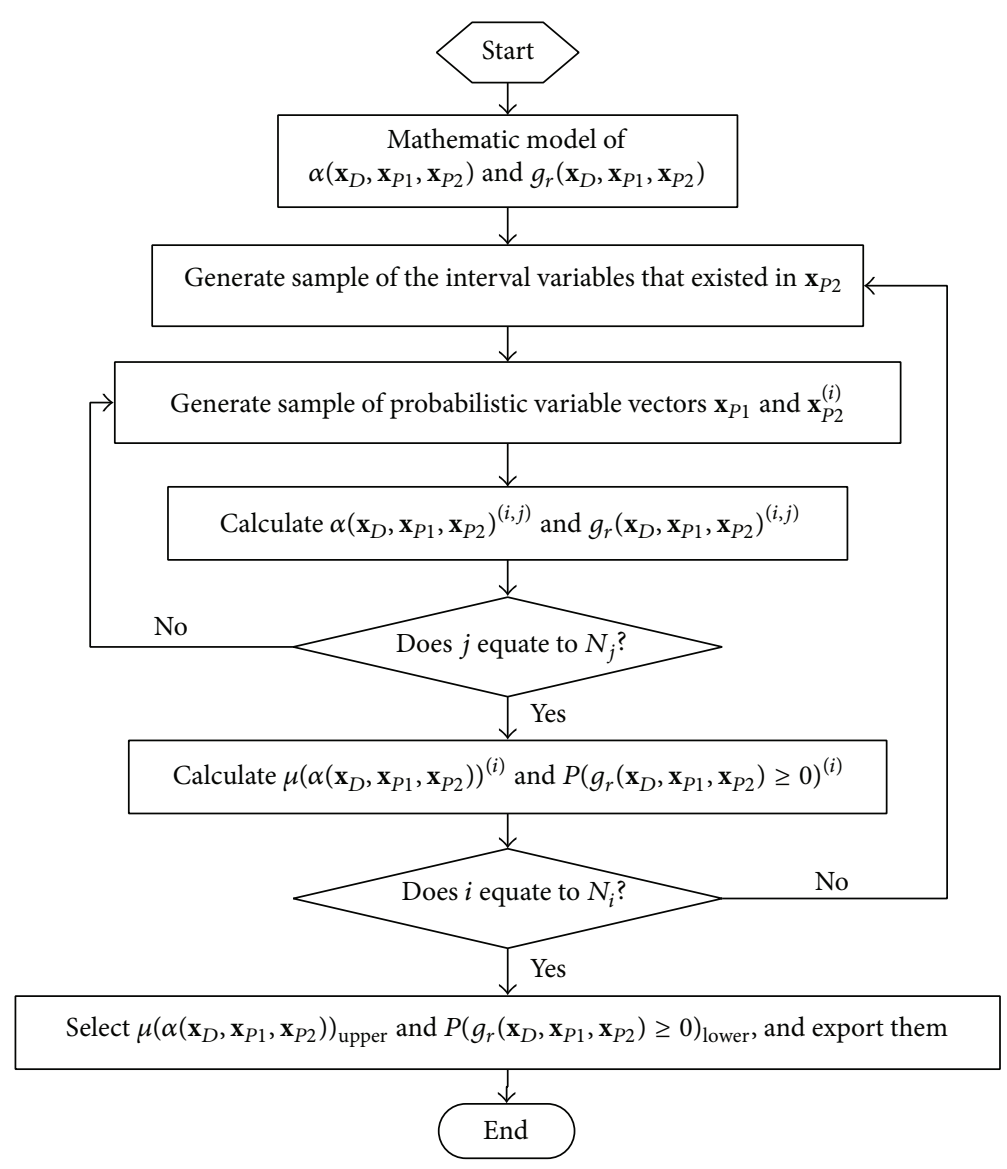

FIGURE 2: Flowchart of the calculation of objective function and constraint function.

\section{The Procedure of the RBDO of the Disc Brake System with Hybrid Uncertainties for Squeal Reduction}

Based on Sections 2 and 3, the main procedure of the RBDO of the disc brake system with hybrid uncertainties for squeal reduction can be summarized as follows.

Step 1. Construct the mathematic model of the RBDO (shown in (17)) for the disc brake system with hybrid uncertainties, based on CEA (shown in (6)) and RSM (shown in (10)).

Step 2. Initialize the design variables $\mathbf{x}_{D}$.

Step 3. Estimate the objective function $\mu\left(\alpha\left(\mathbf{x}_{D}, \mathbf{x}_{P 1}, \mathbf{x}_{P 2}\right)\right)_{\text {upper }}$ and the constrain function $P\left(g_{r}\left(\mathbf{x}_{D}, \mathbf{x}_{P 1}, \mathbf{x}_{P 2}\right) \geq 0\right)_{\text {lower }}$ based on Monte-Carlo method.

Step 4. Is the end condition of the optimization algorithm met? If no, update the design variables and go back to Step 3. If yes, output the optimal design.

The flowchart of the optimization is given in Figure 3.

\section{Numerical Example}

5.1. FE Model of a Simplified Brake. In this section, the simplified disc brake shown in Figure 1 is adopted for investigation and its FE model is shown in Figure 4. The disc is made of grey cast iron which is wear resistant and relatively inexpensive. The friction material is made of anisotropic composite materials and it is mounted to the rigid brake plate which is made of steel.

The FE model is generated by using the three-dimensional continuum elements and the fine meshes are used in the contact areas. The FE model includes 24653 elements and 35685 nodes and the element types are C3D8I and C3D6. The friction contact interactions are defined in the contact surfaces of the pads and the disc. The contact formulations of the pads-to-disc interfaces are "surface-to-surface, smallsliding" with friction and penalty method. The friction at the contact interfaces follows the principle of the Coulomb friction and the contacts follow the principle of hard contacts. The friction and contact formulations in ABAQUS/Standard have been extensively discussed in $[23,32,36]$.

For the constraints and loadings, the disc is rigidly constrained at its five bolt holes and the MOTION card is used to rotate the disc. For the brake pad assemblies, the leading edges are rigidly constrained in the radial direction of the disc 
TABLE 1: The initial values and the ranges of the investigated parameters.

\begin{tabular}{|c|c|c|c|c|}
\hline \multirow{2}{*}{ Parameter } & \multirow{2}{*}{ Unit } & \multirow{2}{*}{ Initial value } & \multicolumn{2}{|c|}{ Range } \\
\hline & & & Lower & Upper \\
\hline Density of back plate $\rho_{1}$ & $\mathrm{~kg} / \mathrm{dm}^{3}$ & 7.82 & 6.50 & 9.00 \\
\hline Elastic modulus of back plate $E_{1}$ & $\mathrm{GPa}$ & 207 & 180 & 230 \\
\hline Thickness of back plate $d_{1}$ & $\mathrm{~mm}$ & 6.20 & 5.00 & 7.50 \\
\hline Density of friction material $\rho_{2}$ & $\mathrm{~kg} / \mathrm{dm}^{3}$ & 2.51 & 2.259 & 2.761 \\
\hline Elastic modulus of friction material $E_{2}$ & $\mathrm{GPa}$ & 5.94 & 4.752 & 7.128 \\
\hline Thickness of friction material $d_{2}$ & $\mathrm{~mm}$ & 12.0 & 8.5 & 12.5 \\
\hline Density of disc $\rho_{3}$ & $\mathrm{~kg} / \mathrm{dm}^{3}$ & 7.20 & 6.48 & 7.92 \\
\hline Elastic modulus of disc $E_{3}$ & $\mathrm{GPa}$ & 125 & 112.5 & 137.5 \\
\hline Thickness of disc $d_{3}$ & $\mathrm{~mm}$ & 20 & 18.5 & 20.5 \\
\hline Brake pressure $p$ & $\mathrm{MPa}$ & 0.5 & 0.45 & 0.55 \\
\hline Friction coefficient $f$ & - & 0.32 & 0.26 & 0.38 \\
\hline
\end{tabular}

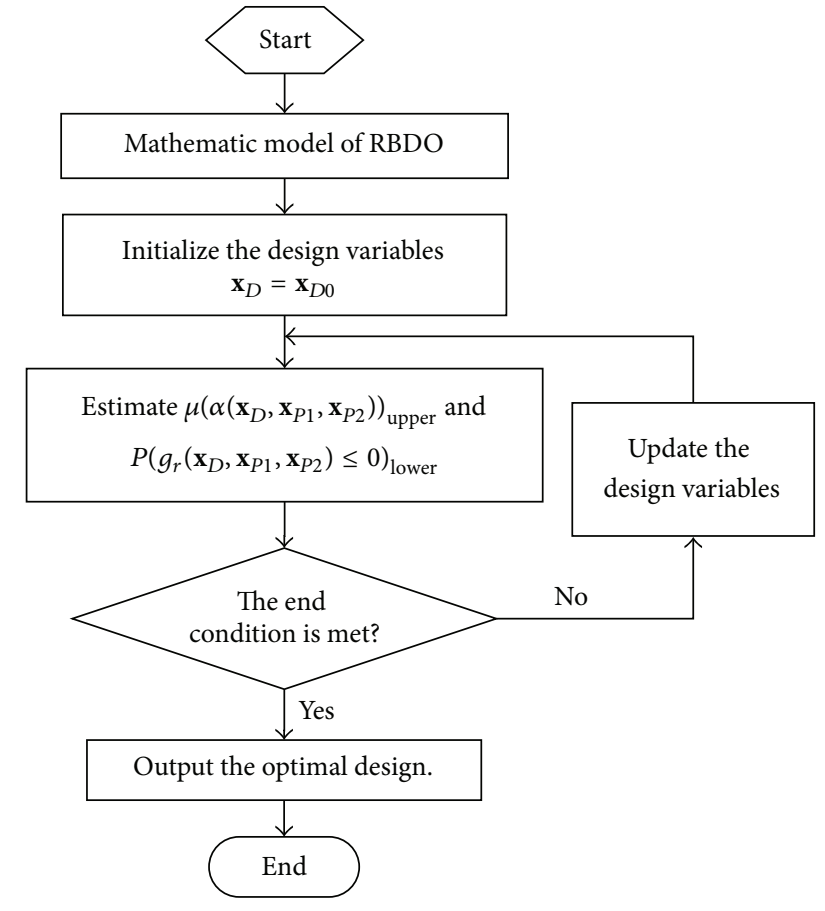

FIGURE 3: Flowchart of the proposed RBDO of the disc brake system with hybrid uncertainties.

while the trailing edges are constrained both at the circumferential and at the radial directions of the disc. The brake pressure is directly applied to the back plates at the contact region between the inner pad and the piston and the contact region between the outer pad and the caliper. It is assumed that an equal magnitude of force acts on each pad assembly.

The main procedure of CEA based on the current FE model contains the following steps: (1) static analysis for the application of brake pressure (0.5 MPa); (2) static analysis to impose the disc's rotational velocity ( $5 \mathrm{rad} / \mathrm{s})$; (3) normal mode analysis to extract the natural frequency to determine the projection subspace; and (4) complex eigenvalue analysis using the subspace projection method.

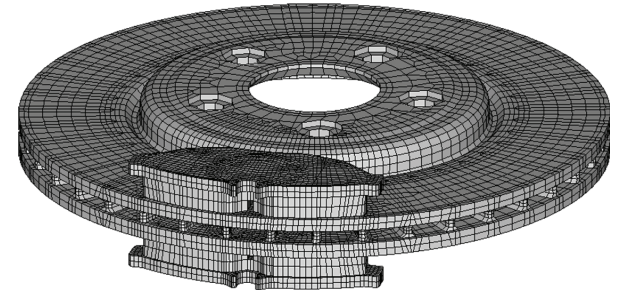

FIgURE 4: The FEM model of a simplified disc brake.

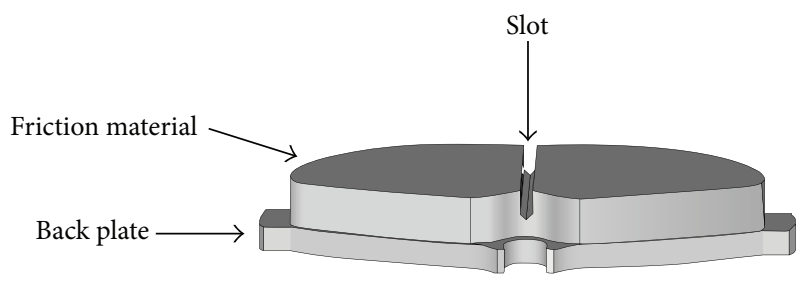

FIgURE 5: The slot of the friction material.

For this commercial brake system, some common and effective measures have been taken to reduce the likelihood of squeal in the early design phase. For example, a slot has been provided in the middle position of the friction material as shown in Figure 5. Therefore, the original stability of this brake is relatively high.

This study aims to optimize the real part of the dominant unstable eigenvalue for reducing potential squeal. The eigenvalue analysis is dependent on a number of factors, such as the stiffness and mass characteristics and operating conditions of the system. Base upon this, the system parameters that we select to investigate are listed in Table 1. The initial values and the variational ranges of the investigated parameters are also listed in Table 1.

In addition, it should be specially pointed out that an assumption of the elastic property for the friction material has been made. For this brake, the friction material is made of organic material and has anisotropic properties. Referring to some earlier works, for instance, the studies of Júnior et al. [34] and Cao et al. [48], we treat the friction material as 


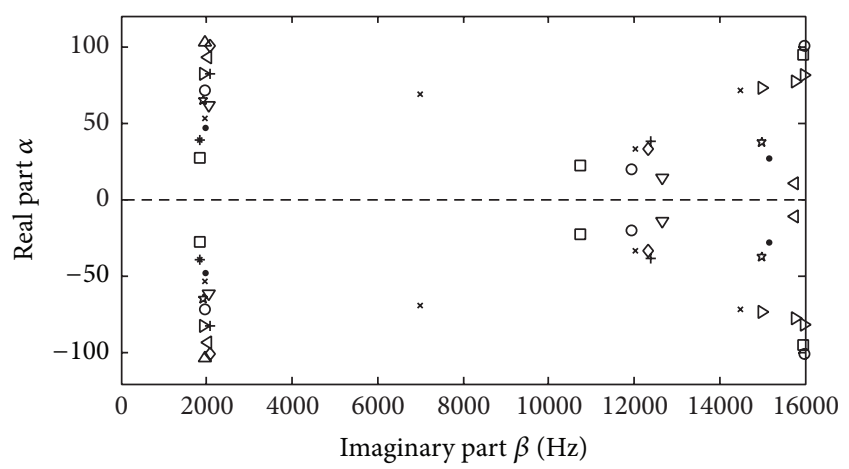

FIgURE 6: The distribution of the complex eigenvalues of the brake system for some samples.

isotropic material but with varied elastic modulus in this research. This assumption can be acceptable in an uncertainty analysis.

5.2. DOE and Determination of Dominant Unstable Eigenvalue. Latin Hypercube Sampling (LHS) method [49] is adopted for DOE, and a total of 120 samples are generated in the design space, which is built up by the parameter variational ranges shown in Table 1. For each sample, CEA is performed in a personal computer based upon (6) and the FE model of the brake. The eigenvalue analysis is carried out in the frequency range of $0-16 \mathrm{kHz}$ where the brake squeal commonly occurs. The analysis results show that the unstable eigenvalues mainly appear around the frequency of $2 \mathrm{kHz}$ in this research. Based on some earlier works, such as the studies of AbuBakar and Ouyang [32] and Nouby et al. [50], we take the unstable eigenvalues at the certain frequency for studying. Therefore, the unstable eigenvalue at $2 \mathrm{kHz}$ for each sample is regarded as the dominant unstable eigenvalue whose real part is positive and is needed to be reduced to improve system stability.

For a clearer understanding, some samples are selected arbitrarily from the 120 samples for investigation, and the complex eigenvalues of the brake system corresponding to these samples are depicted in Figure 6. In the figure, the same markers represent the complex eigenvalues of the system under the same sample. It can be seen that the major unstable frequency is approximately $2 \mathrm{kHz}$.

5.3. Construction of RS Model. Based on the LSM approach and the CEA results of the 120 samples, the RS model of the real part of the dominant unstable eigenvalue is constructed as follows according to (10):

$$
\begin{aligned}
\alpha(\mathbf{x})= & \alpha\left(\rho_{1}, E_{1}, d_{1}, \rho_{2}, E_{2}, d_{2}, \rho_{3}, E_{3}, d_{3}, p, f\right) \\
= & -4173.446+176.689 \rho_{1}-3.495 E_{1}-17.510 d_{1} \\
& +379.791 \rho_{2}+97.618 E_{2}+190.453 d_{2} \\
& +67.872 \rho_{3}-8.742 E_{3}+363.090 d_{3} \\
& -3708.734 p-1801.577 f+0.215 \rho_{1} E_{1}
\end{aligned}
$$

$$
\begin{aligned}
& +2.271 \rho_{1} d_{1}-14.639 \rho_{1} \rho_{2}-4.331 \rho_{1} E_{2} \\
& -3.573 \rho_{1} d_{2}+7.718 \rho_{1} \rho_{3}-0.441 \rho_{1} E_{3} \\
& -8.088 \rho_{1} d_{3}+138.437 \rho_{1} p+90.628 \rho_{1} f \\
& -0.189 E_{1} d_{1}+0.333 E_{1} \rho_{2}+0.036 E_{1} E_{2} \\
& +0.004 E_{1} d_{2}-0.333 E_{1} \rho_{3}+0.014 E_{1} E_{3} \\
& -0.246 E_{1} d_{3}+3.521 E_{1} p+4.897 E_{1} f \\
& -4.135 d_{1} \rho_{2}+7.706 d_{1} E_{2}-1.627 d_{1} d_{2} \\
& -6.547 d_{1} \rho_{3}+0.087 d_{1} E_{3}+4.281 d_{1} d_{3} \\
& -113.832 d_{1} p-56.802 d_{1} f-5.779 \rho_{2} E_{2} \\
& -11.839 \rho_{2} d_{2}-16.931 \rho_{2} \rho_{3}+1.285 \rho_{2} E_{3} \\
& -21.037 \rho_{2} d_{3}+206.002 \rho_{2} p+191.034 \rho_{2} f \\
& -0.562 E_{2} d_{2}+2.141 E_{2} \rho_{3}-0.365 E_{2} E_{3} \\
& -2.088 E_{2} d_{3}-48.563 E_{2} p-29.144 E_{2} f \\
& +0.682 d_{2} \rho_{3}-0.143 d_{2} E_{3}-4.198 d_{2} d_{3} \\
& +18.731 d_{2} p-3.496 d_{2} f+0.415 \rho_{3} E_{3} \\
& +4.098 \rho_{3} d_{3}-102.687 \rho_{3} p+30.748 \rho_{3} f \\
& +0.249 E_{3} d_{3}+3.491 E_{3} p-0.922 E_{3} f \\
& -6.233 d_{3} p-54.917 d_{3} f+2384.332 p f \\
& -3.479 \rho_{1}^{2}+0.009 E_{1}^{2}+3.267 d_{1}^{2}+14.705 \rho_{2}^{2} \\
& +0.475 E_{2}^{2}-0.959 d_{2}^{2}-8.346 \rho_{3}^{2}+0.004 E_{3}^{2} \\
& -5.056 d_{3}^{2}+1797.920 p^{2}+376.181 f^{2} \\
& -10
\end{aligned}
$$

where $\mathbf{x}=\left[\rho_{1}, E_{1}, d_{1}, \rho_{2}, E_{2}, d_{2}, \rho_{3}, E_{3}, d_{3}, p, f\right]^{T}$ is the vector of investigated parameters and $\alpha(\mathbf{x})$ is the real part of the dominant unstable eigenvalue.

It is critical to validate the significance and adequacy of the RS model $\alpha(\mathbf{x})$. For this purpose, ANOVA is carried out and the analysis results are shown in Table 2.

The coefficient of determination $\left(R^{2}\right)$ is defined as the ratio of the explained variable to the total variation. It is a measurement of the degree of fitness. When $R^{2}$ is close to 1 , the model fits the actual experimental data better. The suggested $R^{2}$ value should be at least 0.80 for a good fit of a model [51]. It can be seen from Table 2 that the $R^{2}$ value of $\alpha(\mathbf{x})$ is higher than 0.80 , which indicates that $\alpha(\mathbf{x})$ can explain the observed response well. While the $p$ value is lower than 0.01 , it indicates that the model is considered to be statistically significant [49]. It can be seen from Table 2 that the RS model is highly significant, as the $p$ value of the model is less than 0.0001 .

Hence, the RS model $\alpha(\mathbf{x})$ is successful in describing the correlation between the system parameters and the system response. 
TABLE 2: ANOVA results for the RS model $\alpha(\mathbf{x})$.

\begin{tabular}{|c|c|c|c|c|c|}
\hline Source & Sum of squares & Degree of freedom & Mean square & $F$ value & $p$ value \\
\hline Regression & $1.028 e 5$ & 77 & 1335.03 & 39.68 & $<0.0001$ \\
\hline Residuals & 1412.95 & 42 & 33.64 & & \\
\hline \multirow[t]{2}{*}{ Total } & $1.042 e 5$ & 119 & & & \\
\hline & $R=0.9932$ & & $R^{2}=0.9864$ & & \\
\hline
\end{tabular}

TABLE 3: The probability distributions of probabilistic variables.

\begin{tabular}{|c|c|c|c|c|}
\hline \multirow{2}{*}{ Probabilistic variable } & \multirow{2}{*}{ Unit } & \multirow{2}{*}{ Distribution type } & \multicolumn{2}{|c|}{ Distribution parameters } \\
\hline & & & Expectation $\mu$ & Standard deviation $\sigma$ \\
\hline Density of friction material $\rho_{2}$ & $\mathrm{~kg} / \mathrm{dm}^{3}$ & Normal & 2.51 & 0.04 \\
\hline Elastic modulus of friction material $E_{2}$ & $\mathrm{GPa}$ & Normal & 5.94 & 0.09 \\
\hline Density of disc $\rho_{3}$ & $\mathrm{~kg} / \mathrm{dm}^{3}$ & Normal & 7.2 & 0.10 \\
\hline Elastic modulus of disc $E_{3}$ & $\mathrm{GPa}$ & Normal & 125 & 2.0 \\
\hline Brake pressure $p$ & $\mathrm{MPa}$ & Normal & 0.5 & 0.008 \\
\hline
\end{tabular}

TABLE 4: The probability distributions of interval probabilistic variables.

\begin{tabular}{lcccc}
\hline Interval probabilistic parameter & Unit & Distribution type & \multicolumn{2}{c}{ Distribution parameters } \\
& & & Expectation $\mu$ & Standard deviation $\sigma$ \\
\hline Thickness of friction material $d_{2}$ & $\mathrm{~mm}$ & Normal & {$[9,12]$} & 0.1 \\
Thickness of disc $d_{3}$ & $\mathrm{~mm}$ & Normal & {$[19,20]$} & 0.1 \\
Friction coefficient $f$ & - & {$[0.28,0.36]$} & 0.005 \\
\hline
\end{tabular}

5.4. Uncertainties Existing in the Disc Brake. In practice, uncertainties in material properties, geometry properties, and loading properties are unavoidable. Uncertainties widely exist in a real disc brake system as well. In order to model the uncertainties of the disc brake system more accurately, the uncertain parameters of the brake system with enough information to construct the precise probability distributions are treated as probabilistic variables, while the uncertain parameters with limited data are treated as interval probabilistic variables. Namely, the uncertain variables of the disc brake system are divided into probabilistic variables $\mathbf{x}_{P 1}$ and interval probabilistic variables $\mathbf{x}_{P 2}$.

According to the engineering practice, the density and the elastic modulus of the friction material, the density and the elastic modulus of the disc, and the brake pressure are considered as probabilistic variables; namely, $\mathbf{x}_{P 1}=$ $\left[\rho_{2}, E_{2}, \rho_{3}, E_{3}, p\right]^{T}$. Their probability distributions are listed in Table 3 .

The disc brake system is a complex system related to frictional contact and wear. In the process of braking, the friction coefficient is changed with respect to the sliding velocity between the pad and disc, and the thicknesses of friction material and disc decreases continuously due to wear. Therefore, it is difficult or even impossible to obtain the precise values of the probability distribution parameters of the friction coefficient, the thickness of friction material, and the thickness of disc. As a consequence, these uncertain parameters are assumed as interval probabilistic variables, namely, $\mathbf{x}_{P 2}=\left[d_{2}, d_{3}, f\right]^{T}$. Their probability distributions are listed in Table 4.
5.5. The RBDO of the Disc Brake with Hybrid Uncertainties. The back plate of the brake is considered as the design component and its density, elastic modulus, and thickness parameters are taken as the design variables; namely, $\mathbf{x}_{D}=\left[\rho_{1}, E_{1}\right.$, $\left.d_{1}\right]^{T}$. Due to the manufacturing errors, the actual values of these design variables may deviate from their theoretical values in engineering practice. In this case, $\rho_{1}, E_{1}$, and $d_{1}$ are also treated as probabilistic variables which are assumed to follow normal distributions, and their expectations (marked as $\mu_{\rho_{1}}, \mu_{E_{1}}$, and $\mu_{d_{1}}$ ) are taken as design parameters while their standard deviations (marked as $\sigma_{\rho_{1}}, \sigma_{E_{1}}$, and $\sigma_{d_{1}}$ ) are taken as deterministic values. In this research, the design ranges of $\mu_{\rho_{1}}, \mu_{E_{1}}$, and $\mu_{d_{1}}$ are considered as $7.0 \mathrm{~kg} / \mathrm{dm}^{3} \leq \mu_{\rho_{1}} \leq$ $8.5 \mathrm{~kg} / \mathrm{dm}^{3}, 190 \mathrm{GPa} \leq \mu_{E_{1}} \leq 220 \mathrm{GPa}$, and $5.5 \mathrm{~mm} \leq \mu_{d_{1}} \leq$ $7.0 \mathrm{~mm}$, respectively. The standard deviations of $\mu_{\rho_{1}}, \mu_{E_{1}}$, and $\mu_{d_{1}}$ are considered as $\sigma_{\rho_{1}}=0.1 \mathrm{~kg} / \mathrm{dm}^{3}, \sigma_{E_{1}}=3.0 \mathrm{GPa}$, and $\sigma_{d_{1}}=0.1 \mathrm{~mm}$, respectively.

As is well known, the new brake is usually very stable and the squeal phenomenon commonly occurs in the middle and later stages of using. In order to guarantee the system stability, $\alpha(\mathbf{x})$ should not be larger than the initial deterministic value $\left(\alpha_{0}=81.99\right)$, which can be obtained by the FEA or approximately calculated by the RS model. It can be expressed as $\alpha_{0}-\alpha(\mathbf{x}) \geq 0$. This is taken as one of the constraints for the optimization problem. For lightweight requirement, the mass of the design component should not be larger than the initial value; it can be expressed as $7.82 \times 6.2-\rho_{1} d_{1} \geq 0$. This is taken as the second constraint for the optimization. For 
the requirement of component stiffness, the stiffness of the design component should be taken as one of the constraints as well, which should not be less than the initial value. The back plate's stiffness has a positive correlation with its elastic modulus or its thickness; thus the constraint can be expressed as $E_{1} d_{1}-207 \times 6.2 \geq 0$. This is taken as the third constraint for the optimization.

Based on the discussion mentioned above, the basic mathematical model of the RBDO of the disc brake system with hybrid uncertainties for squeal reduction can be expressed as

$$
\begin{aligned}
\min _{\mu_{\rho_{1}}, \mu_{E_{1}}, \mu_{d_{1}}} & \alpha(\mathbf{x}), \\
\text { s.t. } \quad & P\left(g(\mathbf{x})_{r} \geq 0\right) \geq \eta_{r}, \quad r=1,2,3, \\
& g_{1}(\mathbf{x})=\alpha_{0}-\alpha(\mathbf{x}), \\
& g_{2}(\mathbf{x})=7.82 \times 6.2-\rho_{1} d_{1}, \\
& g_{3}(\mathbf{x})=E_{1} d_{1}-207 \times 6.2, \\
& \mathbf{x}=\left\{\mathbf{x}_{D}, \mathbf{x}_{P 1}, \mathbf{x}_{P 2}\right\}, \\
& \mathbf{x}_{D}=\left[\rho_{1}, E_{1}, d_{1}\right]^{T}, \\
& \mathbf{x}_{P 1}=\left[\rho_{2}, E_{2}, \rho_{3}, E_{3}, p\right]^{T}, \\
& \mathbf{x}_{P 2}=\left[d_{2}, d_{3}, f\right]^{T}, \\
& 7.0 \mathrm{~kg} / \mathrm{dm}^{3} \leq \mu_{\rho_{1}} \leq 8.5 \mathrm{~kg} / \mathrm{dm}^{3}, \\
& 190 \mathrm{GPa} \leq \mu_{E_{1}} \leq 220 \mathrm{GPa}, \\
& 5.5 \mathrm{~mm} \leq \mu_{d_{1}} \leq 7.0 \mathrm{~mm},
\end{aligned}
$$

where $\eta_{r}$ is the design reliability index according to the engineering requirement. $\alpha_{0}$ is the initial value of the real part of the dominant unstable eigenvalue. $\mu_{\rho_{1}}, \mu_{E_{1}}$, and $\mu_{d_{1}}$ are the design parameters. According to Section 3, the constraints $P\left(g(\mathbf{x})_{r} \geq 0\right) \geq \eta_{r}$ can be transformed to $P\left(g_{r}(\mathbf{x}) \geq\right.$ $0)_{\text {lower }} \geq \eta_{r}$, and $\min _{\mu_{\rho_{1}}, \mu_{E_{1}}, \mu_{d_{1}}} \alpha(\mathbf{x})$ can be converted to $\min _{\mu_{\rho_{1}}, \mu_{E_{1}}, \mu_{d_{1}}} \mu(\alpha(\mathbf{x}))_{\text {upper }}$. Therefore, the mathematical model of (19) can be approximated as

$$
\begin{aligned}
\min _{\mu_{\rho_{1}}, \mu_{E_{1}}, \mu_{d_{1}}} & \mu(\alpha(\mathbf{x}))_{\text {upper }}, \\
\text { s.t. } & P\left(g_{r}(\mathbf{x}) \geq 0\right)_{\text {lower }} \geq \eta_{r}, \quad r=1,2,3, \\
& g_{1}(\mathbf{x})=\alpha_{0}-\alpha(\mathbf{x}), \\
& g_{2}(\mathbf{x})=7.82 \times 6.2-\rho_{1} d_{1}, \\
& g_{3}(\mathbf{x})=E_{1} d_{1}-207 \times 6.2, \\
& \mathbf{x}=\left\{\mathbf{x}_{D}, \mathbf{x}_{P 1}, \mathbf{x}_{P 2}\right\}, \\
& \mathbf{x}_{D}=\left[\rho_{1}, E_{1}, d_{1}\right]^{T}, \\
& \mathbf{x}_{P 1}=\left[\rho_{2}, E_{2}, \rho_{3}, E_{3}, p\right]^{T}, \\
& \mathbf{x}_{P 2}=\left[d_{2}, d_{3}, f\right]^{T},
\end{aligned}
$$

TABLE 5: The values of $\mu(\alpha(\mathbf{x}))_{\text {upper }}$ and $P\left(g_{r}(\mathbf{x}) \geq 0\right)_{\text {lower }}$ at the initial values of the design parameters.

\begin{tabular}{lc}
\hline Function & Calculated result \\
\hline$\mu(\alpha(\mathbf{x}))_{\text {upper }}$ & 92.61 \\
$P\left(g_{r}(\mathbf{x}) \geq 0\right)_{\text {lower }}$ & 0.089 \\
\hline
\end{tabular}

TABLE 6: The comparisons of the values of $\mu(\alpha(\mathbf{x}))_{\text {upper }}$ and $P\left(g_{r}(\mathbf{x}) \geq\right.$ $0)_{\text {lower }}$ at initial and optimal values of the design parameters.

\begin{tabular}{lcc}
\hline Function & At initial values & At optimal values \\
\hline$\mu(\alpha(\mathbf{x}))_{\text {upper }}$ & 92.61 & 66.78 \\
$P\left(g_{r}(\mathbf{x}) \geq 0\right)_{\text {lower }}$ & 0.089 & 0.998 \\
\hline
\end{tabular}

$$
\begin{aligned}
& 7.0 \mathrm{~kg} / \mathrm{dm}^{3} \leq \mu_{\rho_{1}} \leq 8.5 \mathrm{~kg} / \mathrm{dm}^{3}, \\
& 190 \mathrm{GPa} \leq \mu_{E_{1}} \leq 220 \mathrm{GPa}, \\
& 5.5 \mathrm{~mm} \leq \mu_{d_{1}} \leq 7.0 \mathrm{~mm} .
\end{aligned}
$$

The value of the design reliability index $\eta_{r}$ is assumed as $\eta_{1}=\eta_{2}=\eta_{3}=0.997$ and $\alpha_{0}$ is taken as 81.99. $\mu(\alpha(\mathbf{x}))_{\text {upper }}$ and $P\left(g_{r}(\mathbf{x}) \geq 0\right)_{\text {lower }}$ are calculated by the Monte-Carlo method with $10^{7}$ samples $\left(N_{i}=10^{3}, N_{j}=10^{4}\right)$ at the initial values of the design parameters $\left(\mu_{\rho_{1}}=7.82 \mathrm{~kg} / \mathrm{dm}^{3}, \mu_{E_{1}}=207 \mathrm{GPa}\right.$, and $\left.\mu_{d_{1}}=6.2 \mathrm{~mm}\right)$, and the results are listed in Table 5 . Here, $P\left(g_{r}(\mathbf{x}) \geq 0\right)_{\text {lower }}$ takes the lowest value of the values of $P\left(g_{1}(\mathbf{x}) \geq 0\right)_{\text {lower }}, P\left(g_{2}(\mathbf{x}) \geq 0\right)_{\text {lower }}$, and $P\left(g_{3}(\mathbf{x}) \geq 0\right)_{\text {lower }}$.

From Table 5, it can be found that the value of $\mu(\alpha(\mathbf{x}))_{\text {upper }}$ is 92.61, which is larger than the initial deterministic value $\left(\alpha_{0}=81.99\right)$. It indicates that the stability of the disc brake system may deteriorate in the course of using under uncertainties, with the initial values of the design parameters. Furthermore, it can be found that $P\left(g_{r}(\mathbf{x}) \geq 0\right)_{\text {lower }}$ is just 0.089 , which is much less than the design requirement $\left(\eta_{r}=\right.$ 0.997 ) and it indicates that the constraints may be seriously destroyed while the uncertainties of the brake system are taken into account. The stability performance of the brake system needs to be improved by optimization design.

The Genetic Algorithm [52] is selected as the optimization method in the implementation of the proposed approach. The parameter values of the back plate are optimized by the Genetic Algorithm based on the Monte-Carlo method, and the design parameters after optimization are $\mu_{\rho_{1}}=7.00 \mathrm{~kg} / \mathrm{m}^{3}, \mu_{E_{1}}=220 \mathrm{GPa}$, and $\mu_{d_{1}}=6.45 \mathrm{~mm}$.

The comparisons of the values of $\mu(\alpha(\mathbf{x}))_{\text {upper }}$ and $P\left(g_{r}(\mathbf{x}) \geq 0\right)_{\text {lower }}$ at the initial and the optimal values of the design parameters are listed in Table 6.

From Table 6 , we can see that $\mu(\alpha(\mathbf{x}))_{\text {upper }}$ is reduced from 92.61 to 66.78 . Namely, it is reduced by about $27.89 \%$ after optimization. So the stability of the uncertain brake system is improved. Furthermore, it can be found that the reliability of the constraints at the initial design parameters violates the design requirement, while it satisfies the design requirement at the optimal values. Therefore, the proposed RBDO can be considered as an effective approach for stability optimization of the disc brake system with hybrid uncertainties. 
TABLE 7: The comparisons among the deterministic optimization, probabilistic optimization, and the proposed optimization.

\begin{tabular}{|c|c|c|c|c|c|}
\hline \multirow{2}{*}{ Type } & \multicolumn{3}{|c|}{ Design parameters } & \multirow{2}{*}{$\mu(\alpha(\mathbf{x}))_{\text {upper }}$} & \multirow{2}{*}{$P\left(g_{r}(\mathbf{x}) \geq 0\right)_{\text {lower }}$} \\
\hline & $\mu_{\rho_{1}}\left(\mathrm{~kg} / \mathrm{m}^{3}\right)$ & $\mu_{E_{1}}(\mathrm{GPa})$ & $\mu_{d_{1}}(\mathrm{~mm})$ & & \\
\hline Initial values & 7.82 & 207 & 6.20 & 92.61 & 0.089 \\
\hline Deterministic optimization & 7.00 & 220 & 7.00 & 57.10 & 0.287 \\
\hline Probabilistic optimization & 7.00 & 219.4 & 6.64 & 65.00 & 0.977 \\
\hline The proposed optimization & 7.00 & 220 & 6.45 & 66.78 & 0.998 \\
\hline
\end{tabular}

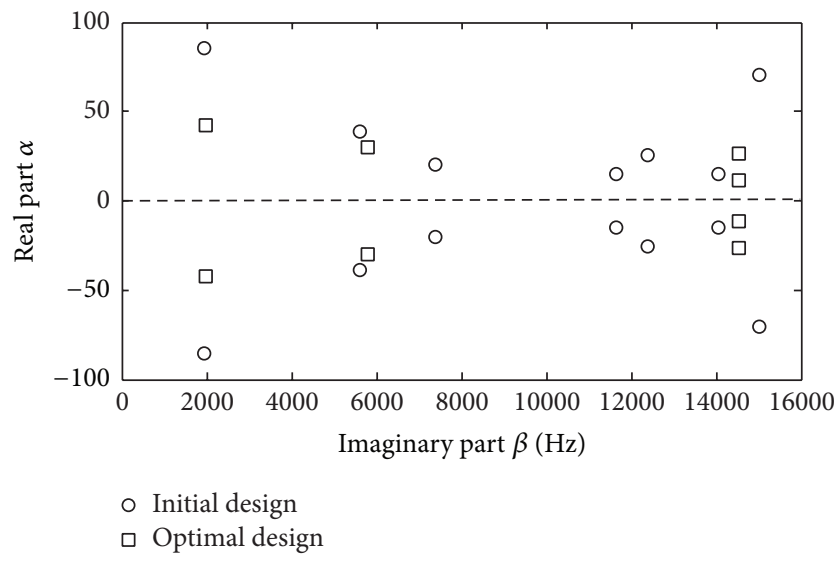

FIgURE 7: The distribution of system complex eigenvalues for initial and optimal designs.

The complex eigenvalues of the brake system at the optimal values of design parameters are plotted in Figure 7. For comparison, the complex eigenvalues of the brake system at the initial values of design parameters are also plotted in Figure 7. The other parameters are all fixed at the initial deterministic values when the design parameters take the initial or the optimal values. It can be seen from Figure 7 that the real part of the dominant unstable eigenvalue of the brake is reduced apparently after optimization. The real parts of the other complex eigenvalues are also reduced. Thus, the system stability is improved.

The comparisons of the system stability at the optimal values of design parameters obtained by the deterministic optimization, the probabilistic optimization, and the proposed optimization are shown in Table 7. When implementing the deterministic optimization, the uncertain variables in this paper are treated as deterministic variables whose values take the initial values of the corresponding parameters. When implementing the probabilistic optimization, the interval probabilistic variables in this paper are treated as probabilistic variables whose expectations take the initial values of the corresponding parameters.

From Table 7, we can find that the optimal values of $\mu(\alpha(\mathbf{x}))_{\text {upper }}$ obtained by the three types of optimizations are all less than the initial value, which means that all these approaches can achieve the goal of optimization. On the other hand, the reliability of the constraints $P\left(g_{r}(\mathbf{x}) \geq 0\right)_{\text {lower }}$ at the optimal values of design parameters obtained by the deterministic optimization is much less than the design requirement $\left(\eta_{r}=0.997\right)$. It indicates that the constraint conditions after optimization may be seriously violated if the uncertainties of the brake system are all neglected. Furthermore, $P\left(g_{r}(\mathbf{x}) \geq 0\right)_{\text {lower }}$ at the optimal values of design parameters obtained by the probabilistic optimization is still failing to achieve the design target. Thus, if we treat the hybrid uncertainties as the probabilistic uncertainties, where the uncertainties of interval variables are neglected, the constraint conditions after optimization may be still violated. However, at the optimal values of design parameters obtained by the proposed optimization, $P\left(g_{r}(\mathbf{x}) \geq 0\right)_{\text {lower }}$ is larger than the design reliability index. Namely, the constraint conditions after optimization are satisfied strictly. Based on the discussion mentioned above, the proposed optimization can be considered as a potential method for squeal reduction of the disc brake system with hybrid uncertainties.

\section{Conclusions}

In engineering applications, uncertainties with and without sufficient information to construct the corresponding precise probability distributions may exist simultaneously. For this case, a hybrid uncertain model is introduced to deal with the stability optimization problem of the disc brake system for squeal reduction in this paper. In the hybrid uncertain model, the thickness of nonwear component, the densities and elastic modulus of component materials, and the brake pressure are treated as probabilistic variables, while the friction coefficient and the thicknesses of wear components are treated as interval probabilistic variables.

Based on the hybrid uncertain model, a RBDO model for the stability optimization of the uncertain disc system is constructed, in which the upper bound of the expectation of the real part of the domain unstable eigenvalue is taken as the design objective, while the lower bounds of the functions related to system stability, the mass and the stiffness of design component, are adopted as the constraints.

By introducing the proposed approach for the stability optimization of a commercial disc brake system with hybrid uncertainties, the real part of the domain unstable eigenvalue of the system is reduced effectively and the constraint reliability at the optimal values of design parameters satisfies the design requirement well. The numerical results also show that if the hybrid uncertainties existing in the disc brake system are neglected, the optimal results obtained by the common 
optimizations may violate the constraint conditions seriously. The proposed stability optimization of the disc brake system with hybrid uncertainties can be considered as a potential method for squeal reduction.

\section{Conflict of Interests}

The authors declare that there is no conflict of interests regarding the publication of this paper.

\section{Acknowledgment}

The paper is supported by National Natural Science Foundation of China (Grant no. 11572121).

\section{References}

[1] A. Akay, "Acoustics of friction," Journal of the Acoustical Society of America, vol. 111, no. 4, pp. 1525-1548, 2002.

[2] R. T. Spurr, "A theory of brake squeal," Proceedings of the Institution of Mechanical Engineers: Automobile Division, vol. 15, no. 1, pp. 33-52, 1961.

[3] S. K. Rhee, P. H. S. Tsang, and Y. S. Wang, "Friction-induced noise and vibration of disc brakes," Wear, vol. 133, no. 1, pp. 3945, 1989.

[4] N. M. Ghazaly, I. Ahmed, and M. El-Sharkawy, "A review of automotive brake squeal mechanisms," Journal of Mechanical Design and Vibration, vol. 1, no. 1, pp. 5-9, 2014.

[5] G. X. Chen and Z. R. Zhou, "A self-excited vibration model based on special elastic vibration modes of friction systems and time delays between the normal and friction forces: a new mechanism for squealing noise," Wear, vol. 262, no. 9-10, pp. 1123-1139, 2007.

[6] S. Yang and R. F. Gibson, "Brake vibration and noise: reviews, comments, and proposals," International Journal of Materials and Product Technology, vol. 12, no. 4-6, pp. 496-513, 1997.

[7] M. Nishiwaki, "Generalized theory of brake noise," Proceedings of the Institution of Mechanical Engineers, Part D: Journal of Automobile Engineering, vol. 207, no. 3, pp. 195-202, 1993.

[8] A. Papinniemi, J. C. S. Lai, J. Zhao, and L. Loader, "Brake squeal: a literature review," Applied Acoustics, vol. 63, no. 4, pp. 391-400, 2002.

[9] N. M. Kinkaid, O. M. O’Reilly, and P. Papadopoulos, "Automotive disc brake squeal," Journal of Sound and Vibration, vol. 267, no. 1, pp. 105-166, 2003.

[10] H. Ouyang, W. Nack, Y. Yuan, and F. Chen, "Numerical analysis of automotive disc brake squeal: a review," International Journal of Vehicle Noise and Vibration, vol. 1, no. 3/4, pp. 207-231, 2005.

[11] D. Guan, X. Su, and F. Zhang, "Sensitivity analysis of brake squeal tendency to substructures' modal parameters," Journal of Sound and Vibration, vol. 291, no. 1-2, pp. 72-80, 2006.

[12] G. Spelsberg-Korspeter, "Structural optimization for the avoidance of self-excited vibrations based on analytical models," Journal of Sound and Vibration, vol. 329, no. 23, pp. 4829-4840, 2010.

[13] G. Spelsberg-Korspeter, "Eigenvalue optimization against brake squeal: symmetry, mathematical background and experiments," Journal of Sound and Vibration, vol. 331, no. 19, pp. 4259-4268, 2012.
[14] S. Lakkam and S. Koetniyom, "Optimization of constrained layer damping for strain energy minimization of vibrating pads," Songklanakarin Journal of Science and Technology, vol. 34, no. 2, pp. 179-187, 2012.

[15] K. Shintani and H. Azegami, "Shape optimization for suppressing brake squeal," Structural and Multidisciplinary Optimization, vol. 50, no. 6, pp. 1127-1135, 2014.

[16] G. Y. Sun, G. Y. Li, Z. H. Gong, X. Y. Cui, X. J. Yang, and Q. $\mathrm{Li}$, "Multiobjective robust optimization method for drawbead design in sheet metal forming," Materials and Design, vol. 31, no. 4, pp. 1917-1929, 2010.

[17] G. Sun, G. Li, S. Zhou, H. Li, S. Hou, and Q. Li, "Crashworthiness design of vehicle by using multiobjective robust optimization," Structural and Multidisciplinary Optimization, vol. 44, no. 1, pp. 99-110, 2011.

[18] N. D. Lagaros, V. Plevris, and M. Papadrakakis, "Neurocomputing strategies for solving reliability-robust design optimization problems," Engineering Computations, vol. 27, no. 7, pp. 819-840, 2010.

[19] I. Lee, K. K. Choi, and D. Gorsich, "Sensitivity analyses of FORM-based and DRM-based performance measure approach (PMA) for reliability-based design optimization (RBDO)," International Journal for Numerical Methods in Engineering, vol. 82, pp. 26-46, 2010.

[20] H. Yu, F. Gillot, and M. Ichchou, "Reliability based robust design optimization for tuned mass damper in passive vibration control of deterministic/uncertain structures," Journal of Sound and Vibration, vol. 332, no. 9, pp. 2222-2238, 2013.

[21] Z. Chen, H. Qiu, L. Gao, L. Su, and P. Li, "An adaptive decoupling approach for reliability-based design optimization," Computers and Structures, vol. 117, pp. 58-66, 2013.

[22] K. K. Chittepu, "Robustness evaluation of brake systems concerned to squeal noise problem," SAE Paper 2011-26-0059, 2011.

[23] E. Sarrouy, O. Dessombz, and J.-J. Sinou, "Piecewise polynomial chaos expansion with an application to brake squeal of a linear brake system," Journal of Sound and Vibration, vol. 332, no. 3, pp. 577-594, 2013.

[24] T. Tison, A. Heussaff, F. Massa, I. Turpin, and R. F. Nunes, "Improvement in the predictivity of squeal simulations: uncertainty and robustness," Journal of Sound and Vibration, vol. 333, no. 15, pp. 3394-3412, 2014.

[25] H. Lü and D. Yu, "Brake squeal reduction of vehicle disc brake system with interval parameters by uncertain optimization," Journal of Sound and Vibration, vol. 333, no. 26, pp. 7313-7325, 2014.

[26] H. Lü and D. Yu, "Stability analysis and improvement of uncertain disk brake systems with random and interval parameters for squeal reduction," Journal of Vibration and Acoustics, vol. 137, no. 5, Article ID 051003, 11 pages, 2015.

[27] G. Stefanou, "The stochastic finite element method: past, present and future," Computer Methods in Applied Mechanics and Engineering, vol. 198, no. 9-12, pp. 1031-1051, 2009.

[28] I. Elishakoff and P. Colombi, "Combination of probabilistic and convex models of uncertainty when scarce knowledge is present on acoustic excitation parameters," Computer Methods in Applied Mechanics and Engineering, vol. 104, no. 2, pp. 187209, 1993.

[29] I. Elishakoff, G. Q. Cai, and J. H. Starnes, "Non-linear buckling of a column with initial imperfections via stochastic and nonstochastic convex models," International Journal of Non-Linear Mechanics, vol. 29, no. 1, pp. 71-82, 1994. 
[30] L. P. Zhu and I. Elishakoff, "Hybrid probabilistic and convex modeling of excitation and response of periodic structures," Mathematical Problems in Engineering, vol. 2, no. 2, pp. 143-163, 1996.

[31] Z. P. Mourelatos and J. Zhou, "A design optimization method using evidence theory," Journal of Mechanical Design, vol. 128, no. 4, pp. 1153-1161, 2006.

[32] A. R. AbuBakar and H. Ouyang, "Complex eigenvalue analysis and dynamic transient analysis in predicting disc brake squeal," International Journal of Vehicle Noise and Vibration, vol. 2, no. 2, pp. 143-155, 2006.

[33] P. Liu, H. Zheng, C. Cai et al., "Analysis of disc brake squeal using the complex eigenvalue method," Applied Acoustics, vol. 68, no. 6, pp. 603-615, 2007.

[34] M. T. Júnior, S. N. Y. Gerges, and R. Jordan, "Analysis of brake squeal noise using the finite element method: a parametric study," Applied Acoustics, vol. 69, no. 2, pp. 147-162, 2008.

[35] N. Coudeyras, J.-J. Sinou, and S. Nacivet, "A new treatment for predicting the self-excited vibrations of nonlinear systems with frictional interfaces: the Constrained Harmonic Balance Method, with application to disc brake squeal," Journal of Sound and Vibration, vol. 319, no. 3-5, pp. 1175-1199, 2009.

[36] A. Nobari, H. Ouyang, and P. Bannister, "Uncertainty quantification of squeal instability via surrogate modelling," Mechanical Systems and Signal Processing, vol. 60-61, pp. 887-908, 2015.

[37] G. Fritz, J.-J. Sinou, J.-M. Duffal, and L. Jézéquel, "Effects of damping on brake squeal coalescence patterns-application on a finite element model," Mechanics Research Communications, vol. 34, no. 2, pp. 181-190, 2007.

[38] J.-J. Sinou, "Transient non-linear dynamic analysis of automotive disc brake squeal-on the need to consider both stability and non-linear analysis," Mechanics Research Communications, vol. 37, no. 1, pp. 96-105, 2010.

[39] J.-J. Sinou, A. Loyer, O. Chiello et al., "A global strategy based on experiments and simulations for squeal prediction on industrial railway brakes," Journal of Sound and Vibration, vol. 332, no. 20, pp. 5068-5085, 2013.

[40] S. Oberst and J. C. S. Lai, "Nonlinear transient and chaotic interactions in disc brake squeal," Journal of Sound and Vibration, vol. 342, pp. 272-289, 2015.

[41] F. Chevillot, J.-J. Sinou, N. Hardouin, and L. Jézéquel, "Effects of damping on the speed of increase and amplitude of limit cycle for an aircraft braking system subjected to mode-coupling instability," Archive of Applied Mechanics, vol. 80, no. 9, pp. 10451054, 2010.

[42] E. Sarrouy, O. Dessombz, and J.-J. Sinou, "Stochastic study of a non-linear self-excited system with friction," European Journal of Mechanics-A/Solids, vol. 40, pp. 1-10, 2013.

[43] J. Fang, Y. Gao, G. Sun, and Q. Li, "Multiobjective reliabilitybased optimization for design of a vehicle door," Finite Elements in Analysis and Design, vol. 67, pp. 13-21, 2013.

[44] D. Baş and I. H. Boyac1, "Modeling and optimization i: usability of response surface methodology," Journal of Food Engineering, vol. 78, no. 3, pp. 836-845, 2007.

[45] R. H. Mayers and D. C. Montgomery, Response Surface Methodology: Process and Product Optimization Using Designed Experiments, John Wiley and Sons, Hoboken, NJ, USA, 2002.

[46] L. Nechak, F. Gillot, S. Besset, and J. J. Sinou, "Sensitivity analysis and Kriging based models for robust stability analysis of brake systems," Mechanics Research Communications, vol. 69, pp. 136$145,2015$.
[47] A. R. AbuBakar and H. Ouyang, "Wear prediction of friction material and brake squeal using the finite element method," Wear, vol. 264, no. 11-12, pp. 1069-1076, 2008.

[48] Q. Cao, H. Ouyang, M. I. Friswell, and J. E. Mottershead, "Linear eigenvalue analysis of the disc-brake squeal problem," International Journal for Numerical Methods in Engineering, vol. 61, no. 9, pp. 1546-1563, 2004.

[49] M. Papila, Accuracy of response surface approximations for weight equations based on structural optimization [Ph.D. thesis], University of Florida, Gainesville, Fla, USA, 2001.

[50] M. Nouby, D. Mathivanan, and K. Srinivasan, "A combined approach of complex eigenvalue analysis and design of experiments (DOE) to study disc brake squeal," International Journal of Engineering, Science and Technology, vol. 1, no. 1, pp. 254-271, 2010.

[51] J. Fu, Y. Zhao, and Q. Wu, "Optimising photoelectrocatalytic oxidation of fulvic acid using response surface methodology," Journal of Hazardous Materials, vol. 144, no. 1-2, pp. 499-505, 2007.

[52] D. E. Goldberg, Genetic Algorithms in Search, Optimization \& Machine Learning, Addison-Wesley, New York, NY, USA, 1989. 


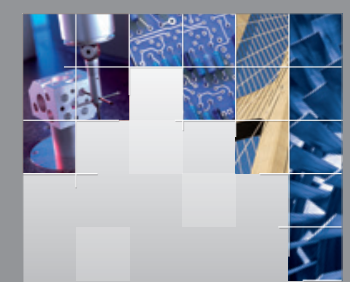

\section{Enfincering}
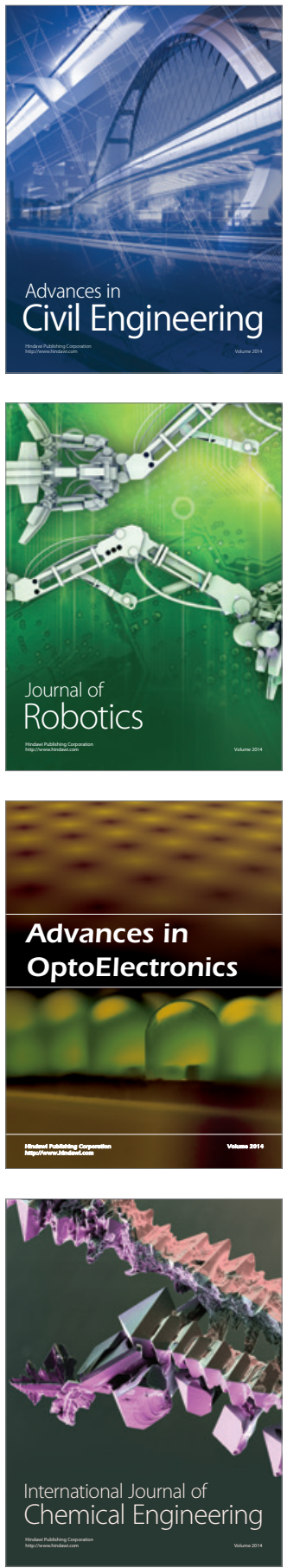

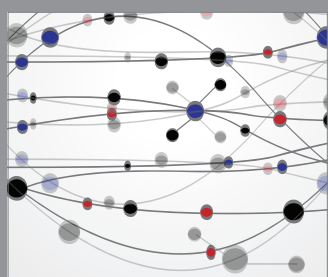

The Scientific World Journal

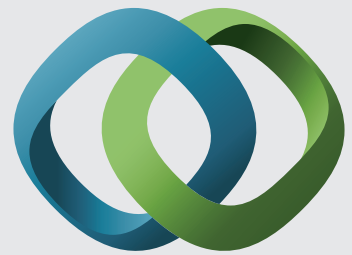

\section{Hindawi}

Submit your manuscripts at

http://www.hindawi.com
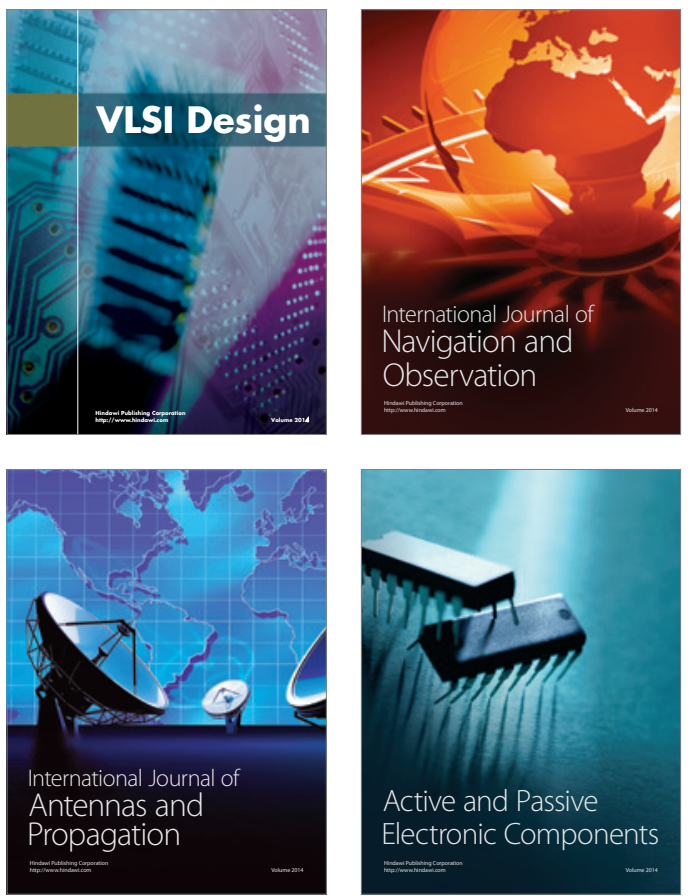
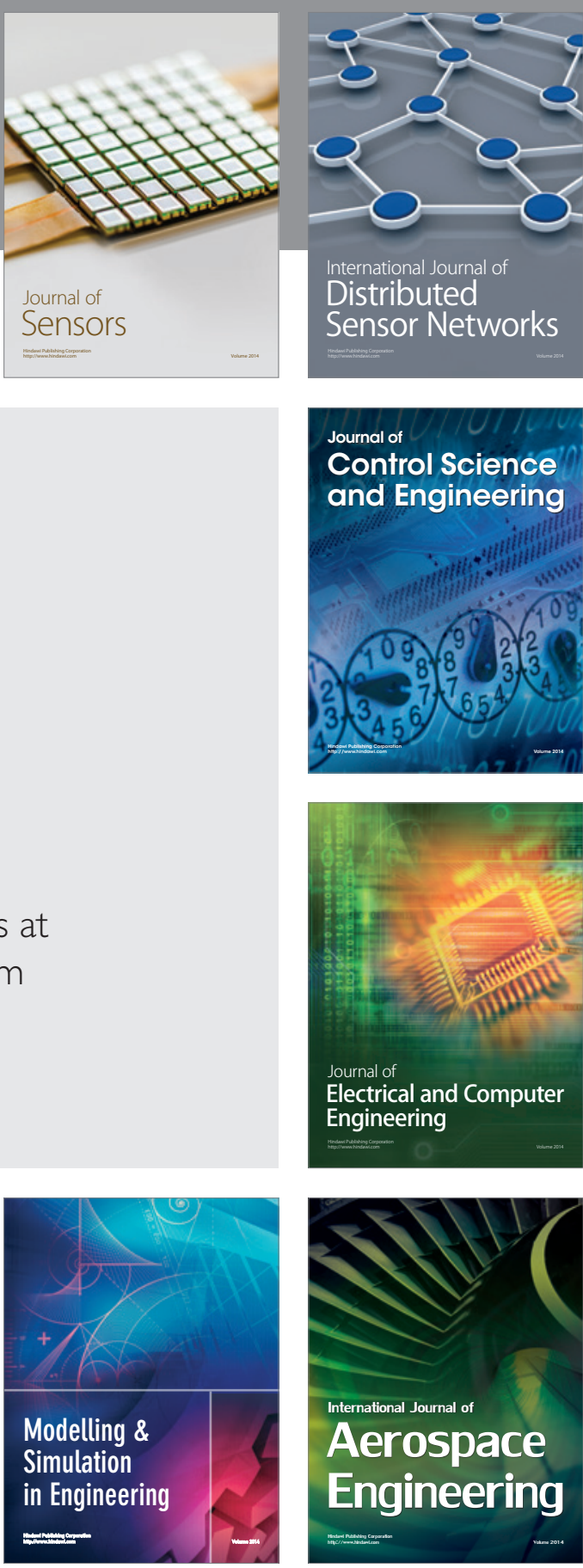

International Journal of

Distributed

Sensor Networks

Journal of

Control Science

and Engineering
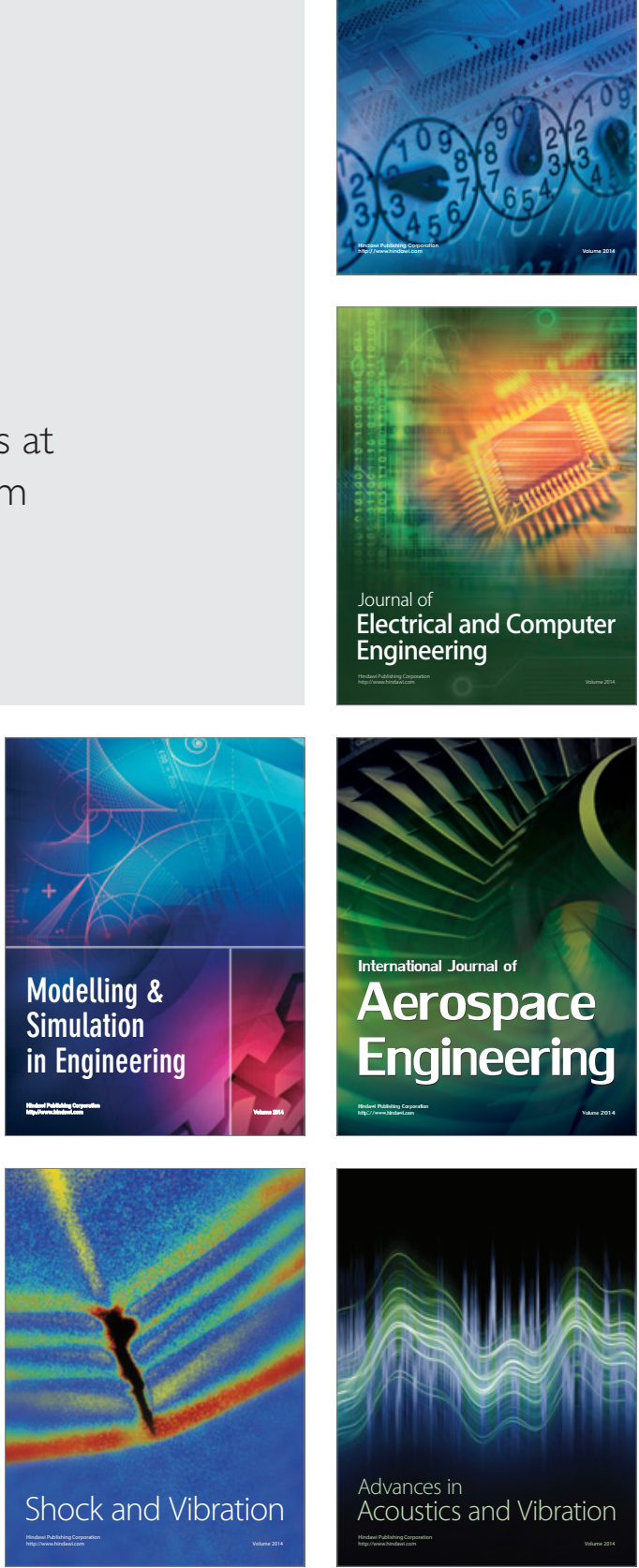